\title{
用扫描隧道显微镜观察 $\mathrm{Cr} 12$ 钢中马氏体
}

\author{
杨志刚 方鸿生王家军 邓旭芯 \\ (清华大学材料科学与工程系, 北京 100084)
}

\section{关键词扫描隧道显微镜 马氏体 孪晶}

扫描隧道显微镜 (STM) 是 80 年代初研制成功的一种新型表面分析仪器 ${ }^{\left[{ }^{[}\right]}$, 现已在物理、化 学、生物等领域获得了广泛应用. 与扫描电子显微镜 (SEM)、透射电子显微镜 (TEM) 及场离 子显微镜 (FIM) 相比, STM 具有结构简单、分辨率高、样品制备方便等特点. STM 的横向分 辨率可超过 $0.1 \mathrm{~nm}$, 纵向分辨率可达 $0.01 \mathrm{~nm}$, 因而 STM 适用于观察样品表面微观结构以及由 于微观缺陷的存在而引起的原子尺度的起伏, 如表面台阶、界面等. 目前 STM 已成功地用于

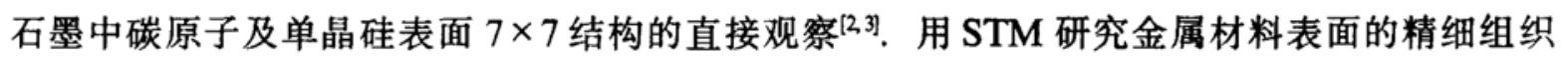
结构, 可有效地填补其它分析手段的不足, 但至今由于实验技术及仪器本身的局限, 扫描隧道 显微镜较少用于工业合金组织的观察与分析. 方鸿生等 ${ }^{[4}$ 曾采用扫描隧道显微镜在大气中研 究了钢中贝氏体的组织结构. 本文首次应用扫描隧道显微镜研究了 $\mathrm{Cr} 12$ 钢中的马氏体组 织.

\section{1 实验材料及试验方法}

实验采用成分为 $\mathrm{Fe}-2.17 \mathrm{C}-12.18 \mathrm{Cr}-0.31 \mathrm{Si}-0.26 \mathrm{Mn}$ (重量百分数) 的合金, 线切割成 $2 \mathrm{~mm}$ 的薄片, 密封于预抽真空的石英管内, 经 $1423 \mathrm{~K}$ 扩散退火 $69 \mathrm{~h}$, 将石英管破碎, 样品淬人室温 水中, 获得奥氏体基体分布着未溶碳化物的组织. 研究表明, 在给定热处理条件下, 合金的 $M_{\mathrm{s}}$ 温度低于零度, 因而, 在水淬过程中, 合金内部不出现马氏体组织. 随后将样品淬人液氮中, 获得马氏体、残余奥氏体及未溶碳化物的组织 (见图 1).

STM 试样尺寸为 $5 \mathrm{~mm} \times 5 \mathrm{~mm} \times 0.7 \mathrm{~mm}$, 试样经预磨抛光后, 采用 $4 \%$ 硝酸酒精溶液在室 温下浸蚀. 经蒸馏水和无水乙醇冲洗后, 立即在样品表面均匀地覆盖一层甘油, 防止样品发 生氧化吸附, 保持表面清洁. 在 STM 实验中, 由于针尖和样品间存在隧道电流, 因此极易吸 引其它杂质、污染表面, 同时样品的氧化也会影响到隧道电流的大小, 从而影响 STM 图象质 量, 所以在大气中进行 STM 试验, 必须对金属样品表面进行保护. 实验中采用偏置电压 $V_{\mathrm{b}}=$ $500 \mathrm{mV}$, 隧道电流 $I=1.0 \mathrm{nA}$, 针尖材料为 $0.5 \mathrm{~mm}$ 直径的铇丝, 铇丝针尖在 $\mathrm{NaOH}$ 溶液中以电 解法制成.

\section{2 实验结果及讨论}

图 1 为扫描电镜中得到的 $\mathrm{Cr} 12$ 钢中马氏体的二次电子图象. 图中白亮块状组织 (箭头

1994-07-28 收稿, 1994-09-26 收修改稿 

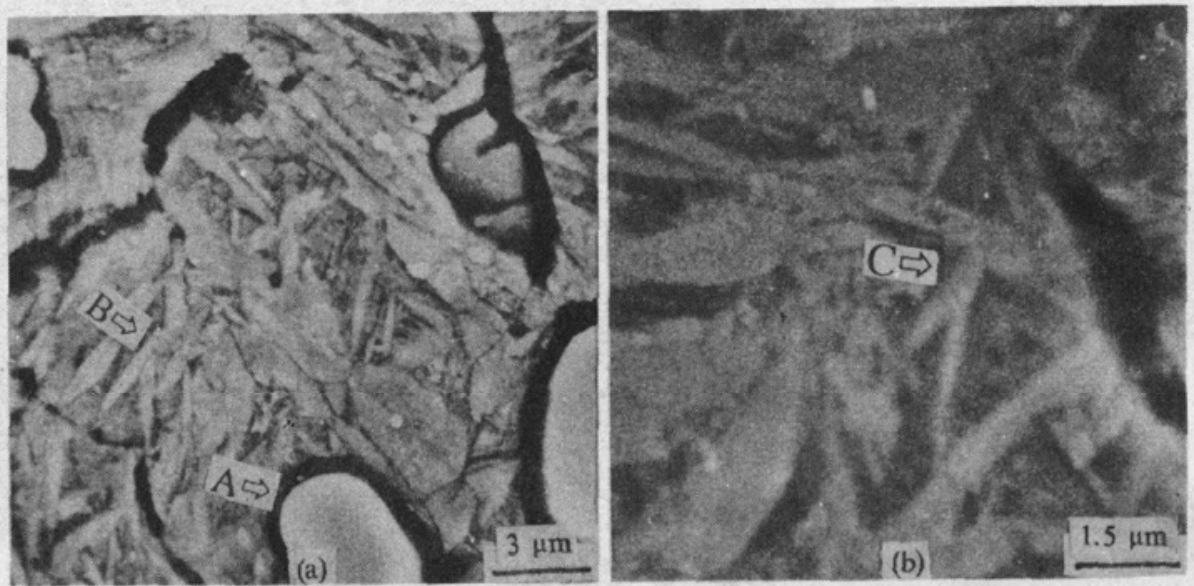

图 $1 \mathrm{Cr} 12$ 钢中马氏体的二次电子象

(a) $\times 5000$. (b) $\times 10000$
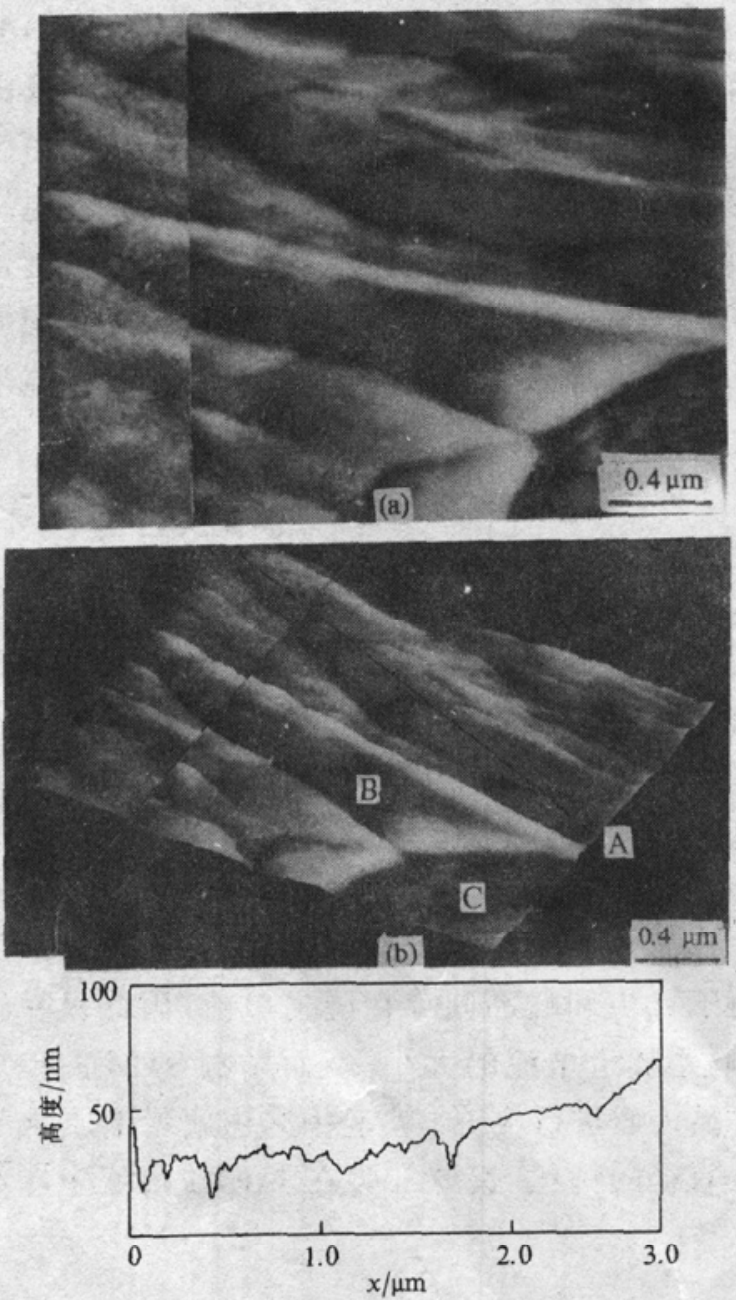

(c)

图 $2 \mathrm{Cr} 12$ 钢中马氏体的 STM 图象

(a)二维形貌图, (b) 三维立体图, (c) 马氏体长轴方向的高度 起伏曲线
A) 为奥氏体化过程中未溶的 $(\mathrm{CrFe})_{7} \mathrm{C}_{3}$ 型碳 化物, 黑色粗线是腐蚀后的奥氏体晶粒边 界, 在灰色奥氏体基体上分布的浅色片条即 为马氏体 (箭头 $\mathrm{B}$ ). 本试验钢在 $1150^{\circ} \mathrm{C}$ 奥 氏体化条件下的 $M_{s}$ 点低于室温, 约为 $-80^{\circ} \mathrm{C}$, 在奥氏体中实际碳含量在 $1.2 \%$ 左 右 ${ }^{[]}$, 经液氮淬火后, 马氏体组织大约占 $70 \%$. 从图 1(a) 中可以看到, 马氏体为典型的高碳 片状马氏体. 马氏体片的尺寸并不均匀, 最 先形成的马氏体片往往横惯整个奥氏体晶 粒, 长度接近 $10 \mu \mathrm{m}$, 宽度可达 $1.5 \mu \mathrm{m}$, 后形 成的马氏体片受到限制, 尺寸较小. 图 1(b) 是 10000 倍下的马氏体的 SEM 图象, 从中 可清楚地看出, 马氏体片之间往往成一定的 角度 (锐角), 马氏体片既可以从奥氏体晶界 处形核长大, 也可以从已形成的马氏体片上 形核长大 (箭头 $\mathrm{C}$ ), 还可以从未溶碳化物处 长大; 较小的马氏体片长约为 $2 \mu \mathrm{m}$, 尖端宽 度约 $0.1 \mu \mathrm{m}$, 但是由于受 SEM 分辨率的限 制, 不能清楚地进一步观察更小的马氏体和 其内部的结构.

试样经腐蚀后在扫描隧道显微镜下的表 面显微组织如图 2 所示, 这是较大范围扫描 $(2 \mu \mathrm{m} \times 2 \mu \mathrm{m})$ 所收集到的两幅图象叠加而 成的 (这两幅图通过微调样品位置而得 
到), 可见图象的重复性相当好, 可信度较 高. 图中可见到两个马氏体片, 其长度超过 了 $2 \mu \mathrm{m}$, 宽度为 $0.2 \mu \mathrm{m}$ 左右, 且从尖端到根 部宽度逐步增加, 马氏体与奥氏体基体的界 面比较平直, 可以看到其中的一个马氏体片 B 起源于一个低坑处 C( 见图 2(b)), 从低坑的形 态看, 它很可能是碳化物在抛光过程中剥落 后留下的痕迹或者是另一晶粒, 因为马氏体 在晶界或碳化物等缺陷处形核, 能量上最有 利. 这里所观察到的马氏体尺寸与 SEM 观

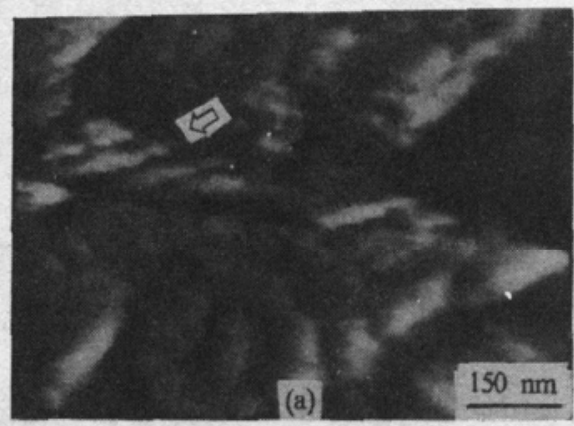
察到的小尺寸的马氏体基本相近 (图 1(b) 中 C), 形态也相似.

STM 还可以给出真实的立体形貌图, 见 图 2(b). 从图 2(b) 中可清楚看出, 两片马氏 体都略高于基体, 而且本身也呈单倾形状. 从图 2(c) 的高度起伏测量线上, 还可以定量 地研究马氏体片上的高度起伏情况, 图 2(c) 是图 2(b) 中一片马氏体片沿长轴 (图 2(b) 中 直线 A 所示) 测量的形貌线, 图中可见有多 条横向的低谷, 说明此处存在线缺陷, 更易于 被腐蚀, 因而高度上有差别(降低了 $10 \mathrm{~nm}$ 左 右), 从其形状及分布分析, 很可能是孪晶

线. 在图 3 中, 这种孪晶线更为典型.

图 3 是 STM 在扫描范围为 $0.8 \mu \mathrm{m} \times$ $0.8 \mu \mathrm{m}$ 时得到的另一片更小的马氏体, 其长 度仅为 $0.8 \mu \mathrm{m}$, 尖端宽度为 $0.04 \mu \mathrm{m}$, 从晶界 界偶处形核, 并沿其中一个晶界长大, 其内部 的孪晶亚结构清晰可辨 (如图中箭头所示). 孪晶规则平行排列, 孪晶线与马氏体长轴呈 $30^{\circ}$ 左右的交角. 由图 3(c) 可见, 孪晶间距为 $40 \mathrm{~nm}$, 孪晶界处腐蚀后高度约降低了 $15 \mathrm{~nm}$.

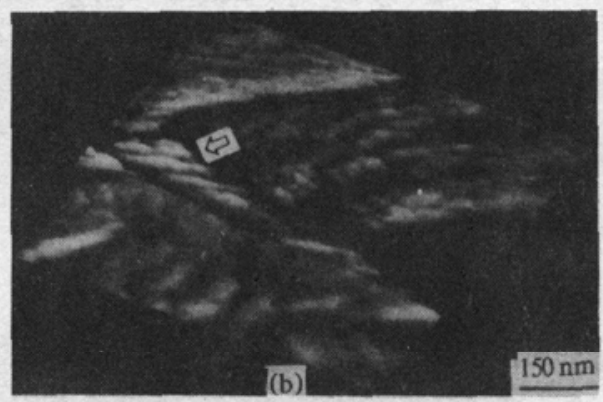

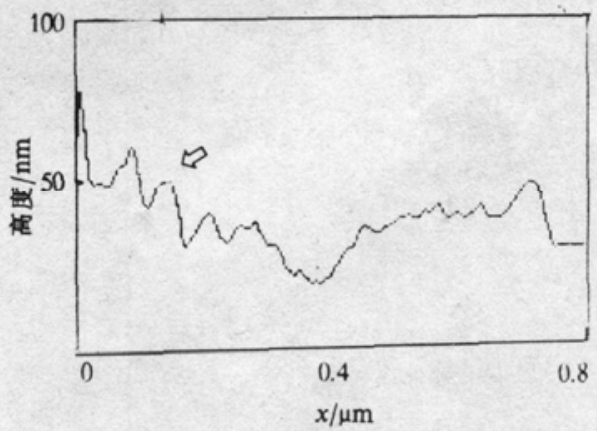

(c)

图 $3 \mathrm{Cr} 12$ 钢中马氏体内孪晶的 STM 图象 (a)二维形貌图, (b) 三维立体图, (c)马氏体内部孪晶的 高度起伏曲线

这里观察到的孪晶的形貌、取向及间距与高碳铬钢中马氏体的透射电镜结果相当符合 ${ }^{[6]}$. 一 般认为, 高碳铬钢中的马氏体的惯习面为 $\{225\}_{\gamma}$, 并在 $\{112\}\langle 111\rangle_{\alpha}$ 系统发生孪生, 根据马氏体 和奥氏体之间的晶体学关系, 此系统等效于奥氏体中的 $\{110\}\langle 110\rangle_{\gamma}$. 图 3 中的孪晶与马氏体 长轴呈 $30^{\circ}$ 交角, 符合上述晶体学关系, 进一步证实了图 3 中的规则排列的薄片为孪晶.

\section{3 结论}

(1) 首次用扫描隧道显微镜在大气中实现了对 $\mathrm{Cr} 12$ 钢液氮淬火后的马氏体组织形态及 
马氏体内的孪晶亚结构的观察;

(2) 在扫描隧道显微镜下观察到了 $\mathrm{Cr} 12$ 钢中马氏体可以从晶界界偶处或未溶碳化物处形 核, 沿晶界长大, 其内部孪晶间距为 $40 \mathrm{~nm}$, 与马氏体长轴呈 $30^{\circ}$ 交角, 属 $\left.\{112\}<111\right\rangle_{\alpha}$ 孪生系 统;

(3) 利用扫描隧道显微镜独具的纵向分辨本领测量了孪晶浸蚀后的高度起伏约为 $15 \mathrm{~nm}$.

\section{参考文献}

1 Binnig G, Rohrer H, Gerher Gh et al. Surface studies by scanning tunneling microscopy. Phys Rev Let, 1982, 49(7): $57 \sim 61$

2 姚朘恩, 商广义, 焦越坎等. 石墨单晶表面原子的扫描隧道显微镜象. 光学学报, 1988, 8(3): 287 288

3 Binnig G, Roherer H, Salvan F et al. Revealing the $7 \times 7$ reconstruction of Si(110). Surface Science, 1985, 157:L373 379

4 方鸿生, 王家军, 杨志刚等. 下贝氏体组织的扫描隧道显微镜研究. 自然科学进展, 1993, 3(6): 525 528

5 方鸿生, 王家军, 郑燕康等. $200 \mathrm{Cr} 12$ 钢下贝氏体碳化物来源及形成机制. 金属学报, 1993, 29(10): A445 450

6 徐祖輝. 马氏体相变与马氏体. 北京: 科学出版社, 1980. 105 112 